\title{
All roads lead to Rome... really?
}

\author{
Song Wan, MD, FRCS
}

\author{
From the Division of Cardiothoracic Surgery, Department of Surgery, The Chinese University of Hong Kong, \\ Prince of Wales Hospital, Hong Kong, China. \\ Disclosures: Author has nothing to disclose with regard to commercial support. \\ Received for publication May 13, 2018; revisions received May 13, 2018; accepted for publication May 14, 2018; \\ available ahead of print June 27, 2018. \\ Address for reprints: Song Wan, MD, FRCS, Division of Cardiothoracic Surgery, The Chinese University of Hong \\ Kong, Prince of Wales Hospital, Hong Kong, China (E-mail: swan@cuhk.edu.hk). \\ J Thorac Cardiovasc Surg 2018;156:1835-6 \\ $0022-5223 / \$ 36.00$ \\ Copyright (c) 2018 by The American Association for Thoracic Surgery \\ https://doi.org/10.1016/j.jtcvs.2018.05.040
}

A seismic shift in the treatment of stenotic aortic valve disease over the past decade has had a marked effect worldwide on the daily practice of cardiac surgeons. A clear reflection of this shift is the rapid popularization of transcatheter aortic valve implantation (TAVI) procedures across Europe and North America. Currently, approximately half of the total number of aortic valve replacement (AVR) procedures in Germany, for instance, are performed via the TAVI approach. Although there is wide recognition that the TAVI approach is a therapeutic advantage in the high-risk patient population, the appropriateness of expanding TAVI indications to intermediate-risk (or even low-risk) patients has recently become a focus of heated debate. Furukawa and colleagues ${ }^{1}$ from Germany are to be congratulated for their timely propensity-matched comparisons of surgical outcomes after minimally invasive AVR and transapical or transfemoral TAVI approaches in this particular patient subgroup. These authors were able to show that the rates of postoperative mortality, stroke, and myocardial infarction were similar among the 3 intermediate-risk groups of patients.

A set of TAVI device (including the valve) is at least 8 to 9 times more expensive than a conventional aortic bioprosthesis. However, the real price to pay may be even higher than what is measured by money alone. Two concerning issues are noteworthy, and their subsequent influence on patients' long-term outcome has yet to be cautiously weighed. First, although postoperative atrial fibrillation was more frequently observed in the minimally invasive AVR group, the patients in the 2 TAVI groups experienced a markedly higher incidence of new-onset complete atrioventricular block, and more of them therefore required permanent pacemaker implantations. Second, a substantially higher incidence rate of paravalvular leakage was found in the 2 TAVI groups. Indeed, these are known complications associated with TAVI intervention and have long been "accepted" when "inoperable" or high-risk patients are concerned. For the intermediate-risk subgroup of patients, however, the unanswered question is: can or should we continue tolerate such high complication rates?

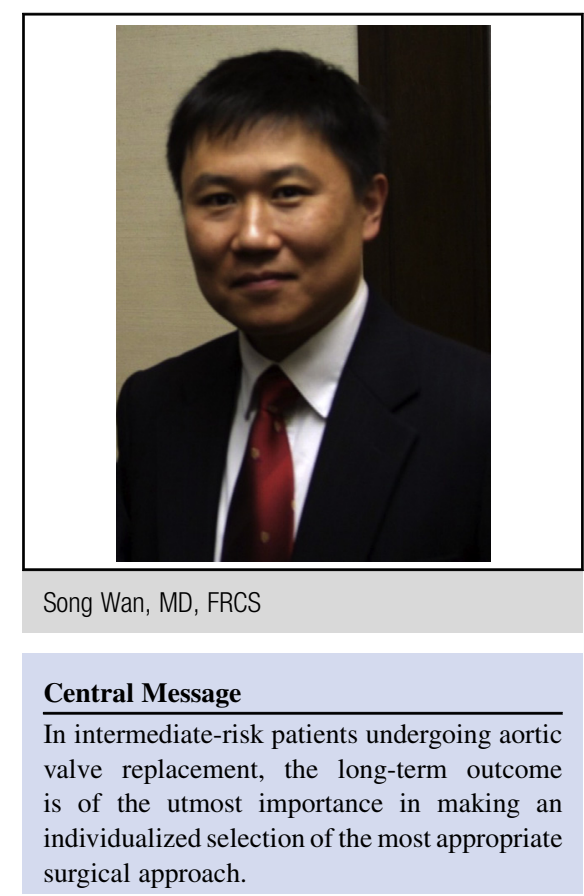

See Article page 1825 .

In fact, Furukawa and colleagues ${ }^{1}$ current report has gone through a thorough peer-review process lasting over a year, with meticulous scrutiny by expert statisticians. Hence, one might not criticize their methodology, namely, the propensity score-matched analysis. Nonetheless, the study end points do remind some readers of the close similarity to numerous earlier investigations such as those on percutaneous coronary intervention versus coronary artery bypass grafting. When only short-term outcome parameters such as mortality, stroke, and myocardial infarction rates were compared, percutaneous coronary intervention was equal to (if not better than) coronary artery bypass grafting in most patients. However, all of those studies have one limitation in common: their conclusions were not fully validated until long-term follow-up data became available from prospective randomized studies with large sample sizes (ie, the Bypass Angioplasty Revascularization Intervention [BARI], Synergy Between Percutaneous Coronary Intervention With TAXUS and Cardiac Surgery [SYNTAX], Future Revascularization Evaluation in Patients With Diabetes Mellitus: Optimal Management of Multivessel Disease [FREEDOM] trials). For the intermediate-risk patients, apart from perioperative complications and short-term clinical outcome, several burning issues are yet to be 
addressed. The durability of prostheses, for example, is top of the list. In this regard, Furukawa and colleagues ${ }^{1}$ did ask an excellent question but were still unable to provide an ultimate answer. Their study indeed leaves more room for us to digest and to judge whether the cup is half full or half empty.

For patients aged 70 years or younger (ie, those in the minimally invasive AVR arm in the current study ${ }^{1}$ ), life expectancy after AVR could be $>10$ years in Western countries. ${ }^{2}$ Unlike the conventional aortic bioprosthesis, which has a proven durability, how long a TAVI valve will truly last is still uncertain. Besides, unless the heavily calcified and stenotic native aortic valve is completely excised, the incidence of atrioventricular block and paravalvular leakage could be difficult to substantially reduce. Hence, caution should always be exercised when considering the choice of surgical AVR or TAVI for intermediate-risk patients.

At the end of the day, although it appears that all roads still lead to Rome, careful measurement is indispensable in finding the best way for an individual traveller. A surgeon's unbiased (evidence-based) advice, as usual, could be life-saving.

\section{References}

1. Furukawa N, Kuss O, Emmel E, Scholtz S, Scholtz W, Fujita B, et al. Minimally invasive versus transapical versus transfemoral aortic valve implantation: a one-to-one-to-one propensity score-matched analysis. J Thorac Cardiovasc Surg. 2018;156:1825-34

2. Rahimtoola SH. Choice of prosthetic heart valve in adults: an update. J Am Coll Cardiol. 2010;55:2413-26. 